031.

\section{The Use of Simulation Software in Developing and Implementing Readiness of Hospitals to Disasterous Situations}

D. Bregman, L. Levi, H. Geva, M. Revach

Acknowledgments to the IDF-Medical Corps, Ministry of

Health and the College of Administration, Tel Aviv, Israel

Theoretically, simulation of disastrous situations has many advantages in preparation of hospital staff to cope with the real scenario. The main problem is to create the database and customizing the software to be friendly, yet realistic.

We describe our experience with developing and implementing the use of simulation software in order to provide perfect drilling technique to be used by Israeli hospitals. The application was developed on SIMAN software. Knowledge and database included the basic disastrous situations, detailed descriptions of casualties, and actual hospital capabilities (staff and infrastructure). A consensus committee decided on crucial model issues and, furthermore, set the thresholds for quality performance indicators.

In phase II, we developed the interfaces to the hospital computer and information systems and updated the various output documentation for each step exercised. Animation proved useful to describe bottle necks in the emergency room, diagnostic departments, and operating rooms. Using this technique in several hospitals of several sizes led to improving the valuable tool further. We currently feel that only lack of resources prohibits us from expanding software to other scenarios of use.

066.

\section{Maxillo-Facial Injuries in Disaster Victims}

A. Limberg, A. Titova, G. Abeleva, I. Fregatov, R. Vreden

Institute of Traumatology and Orthopedics;

Alexander Emergency Hospital, St. Petersburg, Russia

Literature analysis shows a high rate of maxillo-facial injuries (MFI) among victims of transportation and other disasters. They often are overlooked due to their nondominating character, although they deserve special attention, aggravating the body's response to severe trauma.

Analysis of 12,656 badly injured patients with MFI revealed that $98.5 \%$ of them had craniocerebral, $45.1 \%$ ocular, $28.0 \%$ internal chest and abdomen, and $32.2 \%$ locomotive apparatus trauma. The immediate or early specialized treatment for their MFI, alongside life-saving measures was conducted in $95.6 \%$ cases. All of them received orthopedic care; surgical activity amounted to $46.9 \%$. Death rate, in spite of the severity of injuries did not exceed $15 \%$. In $82.1 \%$ of the recovered, the facial form and function were completely restored.

The key to the successful treatment of polytraumatized patients with MFI was in their immediate hospitalization straight to trauma centers having crania-maxillo-facial services; care for MFI as one of shock preventive measures on their arrival; planning the extent and sequence of treatment based on the objective abridged estimation of injury severity score; use of atraumatic, simple, and efficient means of fracture fixation, not interfering with tracheobronchial tree sanitation, tracheal intubation, or brain surgery.

046.

\section{Serum Concentrations of Haptoglobin and Hemoglobin in Massive Blood Transfusions}

Tomoki Nishiyama, $M D, P h D$, Kazuo Hanaoka, $M D, P h D$

Department of Anesthesiology, The University of Tokyo, Faculty of Medicine, Tokyo, Japan

Introduction: In massive blood transfusions, hemolysis is one of the major problems because it sometimes is followed by renal damage. However, there is no detailed report about hemolysis during massive blood transfusions. We studied the relationship between the volume of transfused blood, storage period, and the concentration of hemoglobin $(\mathrm{Hb})$ and haptoglobin (Hp) in patients transfused with $5,000 \mathrm{ml}$ or more of whole blood.

Methods: After the approval by our institute and informed consent from patient's family, 10 patients, 19 to 68 years, were enrolled. The patients in this study received emergency surgery due to multiple trauma or intra-abdominal hemorrhage. Arterial blood was drawn to measure serum concentrations of total and free $\mathrm{Hb}$ and $\mathrm{Hp}$ before the transfusion and at 1,000, 2,000, $3,000,4,000$, and $5,000 \mathrm{ml}$ of transfusion. The stored period for the blood used was monitored. Correlations among these parameters were obtained.

Results: Free $\mathrm{Hb}$ was detected after $2,000 \mathrm{ml}$ transfusion and total $\mathrm{Hb}$ was increased significantly after $3,000 \mathrm{ml}$ transfusion. Total and free $\mathrm{Hp}$ decreased significantly with $1,000 \mathrm{ml}$ transfusion. Total and free Hp had a negative correlation with the total stored period. There was no significant correlation between $\mathrm{Hb}$ and $\mathrm{Hp}$ or between $\mathrm{Hb}$ and stored period.

Conclusion: It was suggested that $1,000 \mathrm{ml}$ or more of transfusion induced the decrease of $\mathrm{Hp}$ and its decrease was accelerated by transfusion of the longer-stored blood, and that 2,000 $\mathrm{ml}$ or more of transfusion resulted in hemolysis.

088.

\section{On the Specificity of the Disaster Medicine Terminology}

M.N. Petkova, $M D, P h D$

Military Medical Academy, Sofia, Bulgaria

This study is a first attempt to analyze the terminology of disaster medicine (DM) in Bulgarian. The terminology is the scientific language that reflects the research, experimental, educational, and applied activities of DM. Because of the specificity of the discipline we use medical terminology, as well as terminology from other fields of knowledge, such as physics. radiobiology, seismology, legislation, planning, management, etc. So as the DM terminology becomes more exact, it experiences an evi- 